\title{
Bestimmung von hydraulischen Parametern in Lockergesteinen: Ein Vergleich unterschiedlicher Feldmethoden
}

\section{Journal Article}

Author(s):

Brauchler, Ralf; Hu, Rui; Hu, Linwei; Ptak, Thomas

Publication date:

2012-06

Permanent link:

https://doi.org/10.3929/ethz-b-000412590

Rights / license:

In Copyright - Non-Commercial Use Permitted

Originally published in:

Grundwasser 17(2), https://doi.org/10.1007/s00767-011-0185-6 


\title{
Bestimmung von hydraulischen Parametern in Lockergesteinen: Ein Vergleich unterschiedlicher Feldmethoden
}

\author{
Ralf Brauchler · Rui Hu • Linwei Hu • Thomas Ptak
}

Eingang des Beitrages: 21.1.2011 / Eingang des überarbeiteten Beitrages: 28.9.2011 / Online veröffentlicht: 19.1.2012

(C) Springer-Verlag 2012

Zusammenfassung In dieser Feldstudie werden die laufzeitbasierte tomographische Inversion von Daten aus Kurzzeitpumpversuchen mit der analytischen Auswertung verglichen und die ermittelten hydraulischen Parameter hinsichtlich ihrer räumlichen Auflösung diskutiert und bewertet. Als Datenbasis dienen Messergebnisse aus Kurzzeitpumpversuchen, die in einer tomographischen Messanordnung in einem zwei Meter mächtigen, gut charakterisierten Sand- und Kiesgrundwasserleiter unter Verwendung eines 2“-Brunnens und eines Multikammerbrunnens, beide mit Direct-Push-Technik installiert, durchgeführt wurden. Die analytische Auswertung der Kurzzeitpumpversuche hat gezeigt, dass es nicht möglich ist, Bereiche mit unterschiedlichen hydraulischen Eigenschaften voneinander abzugrenzen. Entsprechend einem Vergleich mit den Ergebnissen von Multilevel-Slug-Tests werden die ermittelten hydraulischen Parameter, trotz einer geringen Pumpdauer von 200 Sekunden und hydraulisch isolierten Pump- und Beobachtungsintervallen, von einem hydraulisch höher durchlässigen Bereich am unteren Rand des Grundwasserleiters dominiert.

\footnotetext{
Dr. R. Brauchler $(\bowtie)$

Department Erdwissenschaften, Eidgenössische

Technische Hochschule Zürich,

Sonneggstraße 5, 8092 Zürich, Schweiz

E-Mail: ralf.brauchler@erdw.ethz.ch

R. Hu $\cdot$ L. Hu $\cdot$ Prof. Dr.-Ing. T. Ptak

Geowissenschaftliches Zentrum, Universität Göttingen,

Goldschmidtstraße 3, 37077 Göttingen, Deutschland

R. $\mathrm{Hu}$

E-Mail: rhu@uni-goettingen.de

L. Hu

E-Mail: linwei.hu@stud.uni-goettingen.de

Prof. Dr.-Ing. T. Ptak

E-Mail: thomas.ptak@geo.uni-goettingen.de
}

Die laufzeitbasierte tomographische Inversion ermöglicht hingegen, vertikale und laterale Änderungen der Diffusivitätsverteilung zwischen Pump- und Beobachtungsbrunnen hochaufgelöst zu rekonstruieren.

\section{Investigation of hydraulic parameters in unconsolidated sediments: a comparison of methods}

\begin{abstract}
In this study the potential of a hydraulic traveltime based inversion approach with analytical solutions for the evaluation of short term pumping tests is assessed. The data base comprises measurements from short-term pumping tests performed in a sand and gravel aquifer using a tomographic measurement array. The evaluation, which is based on an analytical solution, has shown that it is not possible to delimit aquifer zones with different hydraulic properties. The comparison with multi-level slug tests has revealed that the pumping test results are dominated by a zone with a relatively high hydraulic conductivity located close to the bottom of the aquifer. This finding is surprising due to the short pumping time of 200 seconds and due to the hydraulically isolated pumping and observation intervals. The travel-time based inversion, however, allows the reconstruction of vertical and lateral changes in hydraulic diffusivity, between pumping and observation wells, with a high resolution.
\end{abstract}

Keywords Direct-Push · Multi-level slug tests · Short-term pumping tests $\cdot$ Hydraulic tomography

\section{Einleitung}

Für nahezu jede hydrogeologische Untergrundcharakterisierung werden Kenntnisse über die Parameter hydraulische 
Durchlässigkeit und spezifischer Speicherkoeffizient benötigt. Diese Parameter beschreiben die Strömungs- und Speichereigenschaften des Wassers und des Grundwasserleiters. Der Quotient aus hydraulischer Durchlässigkeit und spezifischem Speicherkoeffizienten wird als Diffusivität bezeichnet und ist ein Maß für die Geschwindigkeit, mit der sich eine Druckänderung in einem Grundwasserleiter ausbreitet. Diese Druckausbreitung als Funktion des Ortes und der Zeit wird mit der Grundwasserströmungsgleichung beschrieben.

Bei der Vorhersage von Stofftransport im Untergrund spielen kleinräumige Heterogenitäten der hydraulischen Durchlässigkeit eine dominierende Rolle (z. B. Sudicky \& Huyakorn 1991, Zheng \& Gorelick 2003, Teutsch et al. 1998). Geologische Erkundungsverfahren wie z. B. Bohrungen stellen allerdings nur punktuelle Aufschlüsse dar, die infolge des Punktcharakters und aufgrund des meist komplexen geologischen Aufbaus strömungs- und transportprozess-relevante Untergrundstrukturen $u$. U. verfehlen oder nicht ausreichend charakterisieren können. Pumpversuche können dagegen hydraulische Parameter über einen größeren Bereich ermitteln, kleinräumige Diskontinuitäten, die den Transport von gelösten Stoffen im Grundwasser signifikant beeinflussen, können jedoch nicht ermittelt werden. Labormethoden wie Permeametermessungen oder Korngrößenanalysen erlauben eine hochaufgelöste Bestimmung von hydraulischen Eigenschaften. Es gibt aber offene Fragen hinsichtlich der Verlässlichkeit von ermittelten hydraulischen Parametern (Klute \& Dirksen 1986, Rovey \& Niemann 1998, Gee \& Bauder 1986, Danielson \& Sutherland 1986). Auch ist der Erkundungsaufwand für eine räumlich hinreichend auflösende Charakterisierung in der Regel limitierend.

Zur hochauflösenden Beschreibung der räumlichen Variation von hydraulischen Parametern in unterschiedlichen Tiefen wurden daher verschiedene hydraulische Methoden entwickelt. Hierbei sind unter anderem "Dipol-Flowtests“ (Kabala 1993, Zlotnik \& Zurbuchen 1998, Zlotnik \& Ledder 1996, Peursem et al. 1999, Zlotnik et al. 2001), FlowmeterTests (Molz et al. 1989, Molz \& Young 1993, Young \& Pearson 1995, Boman et al. 1997) und Multilevel-SlugTests (Melville et al. 1991, Butler et al. 1994, Butler 1998, Brauchler et al. 2010, Diem et al. 2010) zu nennen. Für Lockergesteinsgrundwasserleiter wurden in den letzten Jahren spezielle Sonden entwickelt, die es unter Verwendung von Direct-Push-Technologie (z. B. Dietrich \& Leven 2006) ermöglichen, die hydraulische Durchlässigkeit in unterschiedlichen Tiefen beim Vortrieb hochaufgelöst zu bestimmen (Butler et al. 2007, Dietrich et al. 2008, Liu et al. 2009, Lessoff et al. 2010). Die oben genannten Methoden erlauben es, vertikale Variationen der hydraulischen Durchlässigkeit hochaufgelöst zu bestimmen.

Zur hochaufgelösten Bestimmung von vertikalen und horizontalen Variationen der hydraulischen Durchlässigkeit und des spezifischen Speicherkoeffizienten haben in den letzten 15 Jahren verschiedene Arbeitsgruppen begonnen, Auswerteansätze für die Inversion von hydraulischen Tests, die unter Verwendung einer tomographischen Messanordnung durchgeführt wurden, zu entwickeln. Tomographische Messanordnungen können unter Verwendung von Multipackersystemen und/oder Multikammerbrunnen realisiert werden. Dabei kann durch Variation der Pump- und Beobachtungsintervalle (Brunnenfilterstreckenabschnitte) ein Strömungsmuster erzeugt werden, das mit den Strahlwegen eines seismisch-tomographischen Experiments verglichen werden kann. Aufgrund der tomographischen Messanordnung wird diese Methode in der Literatur als hydraulische Tomographie bezeichnet. Durch die Verwendung von geeigneten Inversionsmodellen kann dann über das Fitting der aufgezeichneten Druckänderungen die hydraulische Parameterverteilung zwischen zwei oder mehr Bohrlöchern sowohl in vertikaler als auch in horizontaler Richtung hochaufgelöst rekonstruiert werden (z. B. Gottlieb \& Dietrich 1995, Yeh \& Liu 2000, Vesselinov et al. 2001a, 2001b, Zhu $\&$ Yeh 2005). Die entwickelten Inversionstechniken wurden mittels Laborexperimenten (McDermott et al. 2003, Illman et al. 2007, 2009, 2010, Liu et al. 2002, 2007) und Felddaten (Bohling et al. 2007, Li et al. 2005, 2007, Straface et al. 2007) erfolgreich getestet.

Eine Alternative zu den oben genannten Inversionsansätzen besteht in der Inversion der Laufzeiten von Druckänderungssignalen. Dieser Inversionsansatz folgt der seismischen Strahltomographie und basiert auf der Transformation der instationären Strömungsgleichung in eine Wellengleichung unter Verwendung eines asymptotischen Ansatzes (Virieux et al. 1994). Die resultierende Eikonalgleichung kann mit „Ray-Tracing“-Techniken oder „ParticleTracking"-Methoden gelöst werden (z. B. Vasco et al. 2000, Vasco \& Karasaki 2006, Kulkarni et al. 2001, Datta-Gupta et al. 2001, He et al. 2006, Brauchler et al. 2003, 2007, 2010, 2011). Die Inversion beruht dabei auf der Lösung eines Laufzeitintegrals, das den Zusammenhang zwischen der Laufzeit des Maximums einer aufgezeichneten instationären hydraulischen Druckänderung und der Diffusivität des untersuchten Systems beschreibt (Vasco et al. 2000, Brauchler et al. 2007, 2010).

Ein großer Vorteil der hydraulischen Tomographie gegenüber der Auswertung basierend auf analytischen Lösungen besteht in der hohen räumlichen Auflösung und der räumlichen Zuordnung der hydraulischen Parameter. Hingegen ist bei der Anwendung von analytischen Lösungen zur Auswertung von hydraulischen Tests mit tomographischer Anordnung die räumliche Zuordnung der ermittelten hydraulischen Parametern immer mit Unsicherheiten behaftet (Leven \& Dietrich 2006), da sie sowohl von der Testkonfiguration als auch von der Heterogenitätsverteilung des untersuchten Mediums (Wu et al. 2005) abhängig ist. 


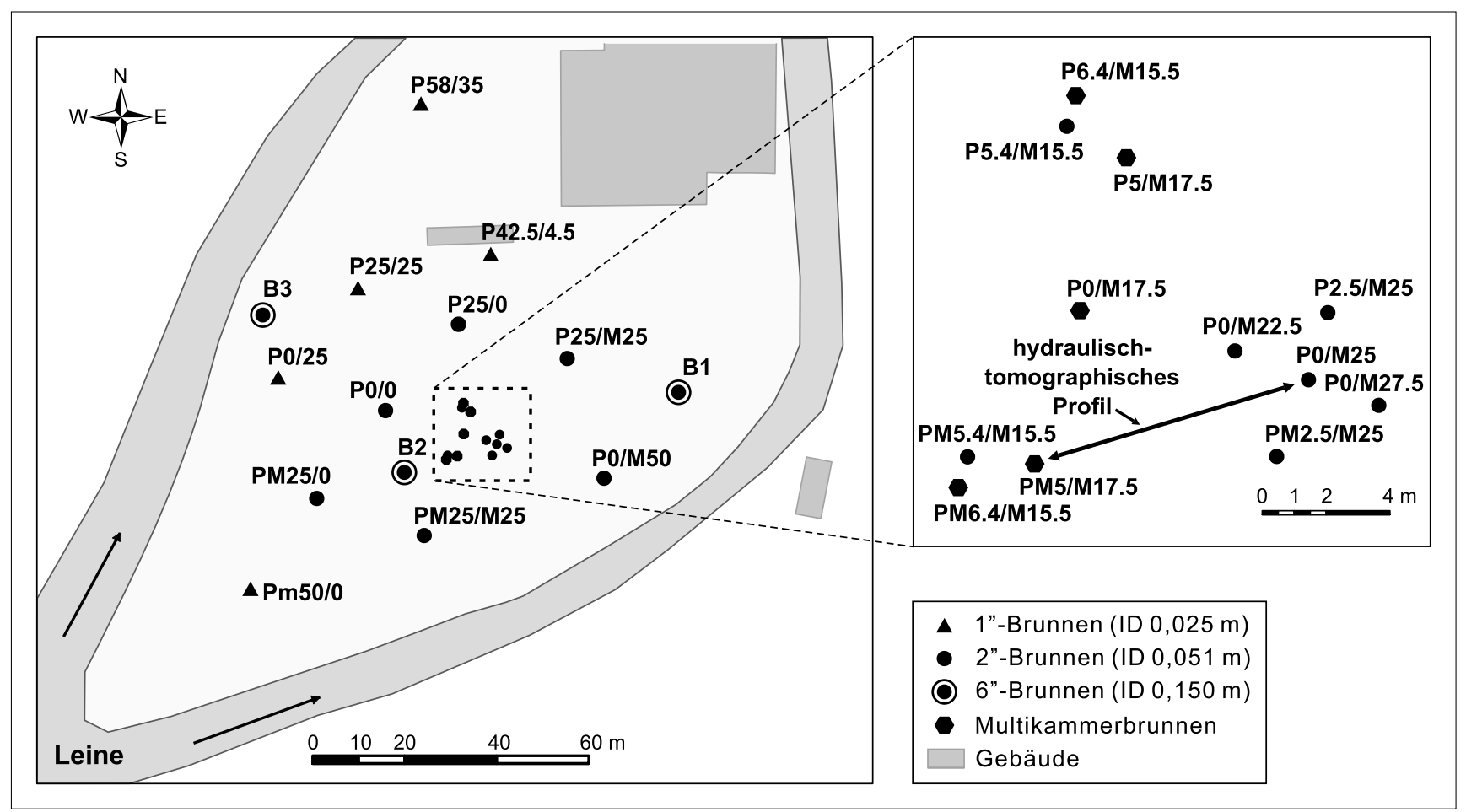

Abb. 1 Lage der Brunnen im Testfeld „Stegemühle“ in unmittelbarer Nähe zum Stadtgebiet Göttingen, Deutschland.

In dieser Feldstudie wird die laufzeitbasierte tomographische Inversion von Daten aus Kurzzeitpumpversuchen mit der analytischen Auswertung verglichen und die ermittelten hydraulischen Parameter hinsichtlich ihrer räumlichen Auflösung diskutiert und bewertet. Darüber hinaus werden Daten aus Multilevel-Slug-Tests zur Interpretation der Ergebnisse herangezogen. Als Datenbasis dienen 30 Absenkungskurven aus Pumpversuchen, die unter Verwendung von zwei $9 \mathrm{~m}$ voneinander entfernten Brunnen durchgeführt wurden. Im Pumpbrunnen wurden unter Verwendung eines Doppelpackersystems an sechs Filterstreckenintervallen, gleichmäBig verteilt über die gesamte Mächtigkeit des Grundwasserleiters, Kurzzeitpumpversuche durchgeführt. Die Druckänderungen wurden dabei jeweils in sechs Tiefen in einem Multikammerbrunnen aufgezeichnet. Diese tomographische Messanordnung ermöglichte es, ein zweidimensionales, räumlich hochaufgelöstes Profil der hydraulischen Diffusivität zu rekonstruieren.

\section{Beschreibung des Testfeldes}

Die vorgestellten hydraulischen Untersuchungen wurden im Testfeld „Stegemühle“ in einem gut charakterisierten Sand- und Kiesgrundwasserleiter im Leinetal, in unmittelbarer Nähe zu Göttingen, durchgeführt. Hierzu wurde in dem Testfeld ein dichtes Messnetz, bestehend aus 1"“-, 2“- und 6"-Messstellen sowie Multikammerbrunnen, eingerichtet. Abbildung 1 zeigt die Lage der Messstellen. Für den Bau der 1"-, 2“- und Multikammerbrunnen wurde Direct-PushTechnik eingesetzt (z. B. Dietrich \& Leven 2006). Der Einsatz der Direct-Push-Technik eignet sich besonders zur Erstellung von Messstellen an denen hydraulische MultilevelVersuche durchgeführt werden. Durch das Kollabieren des Aquifermaterials im gesättigten Bereich, beim Herausziehen des Direct-Push-Gestänges, wird der Bohrlochzwischenraum verschlossen und dadurch die Wahrscheinlichkeit von Umläufigkeiten minimiert. Die 6"-Brunnen wurden mit einem konventionellen Bohrgerät erstellt.

Der strukturelle Aufbau des Grundwasserleiters, d. h. Ober- und Unterkante des Grundwasserleiters, wurde über elektrische Leitfähigkeitsprofile, die mit einer Direct-PushLeitfähigkeitssonde aufgezeichnet wurden, bestimmt (z. B. Christy et al. 1994, Schulmeister et al. 2003). Abbildung 2 zeigt exemplarisch den Schichtaufbau im Bereich des 2"-Brunnens (P0/M25) und des Multikammerbrunnen (PM6.4/M15.5). Die Interpretation basiert auf den elektrischen Leitfähigkeitsprofilen und Bohrkerninformationen. Wie die geologische Interpretation zeigt, bestehen die Lockersedimente aus einer ca. 3,5 m mächtigen Silt- und Tonschicht, die den eigentlichen Grundwasserleiter überlagert. Der Grundwasserleiter besteht aus Sand und Kies mit einer durchschnittlichen Mächtigkeit von 2,5 m. Die Grundwasserleiterunterkante bildet Tonstein des mittleren Keupers. Ein Vergleich der elektrischen Leitfähigkeitspro- 
Abb. 2 Geologische Interpretation des oberflächennahen Untergrunds basierend auf Direct-Push-Leitfähigkeitsprofilen am Feldstandort ,Stegemühle“ a) DP-Leitfähigkeitslog P0/M25

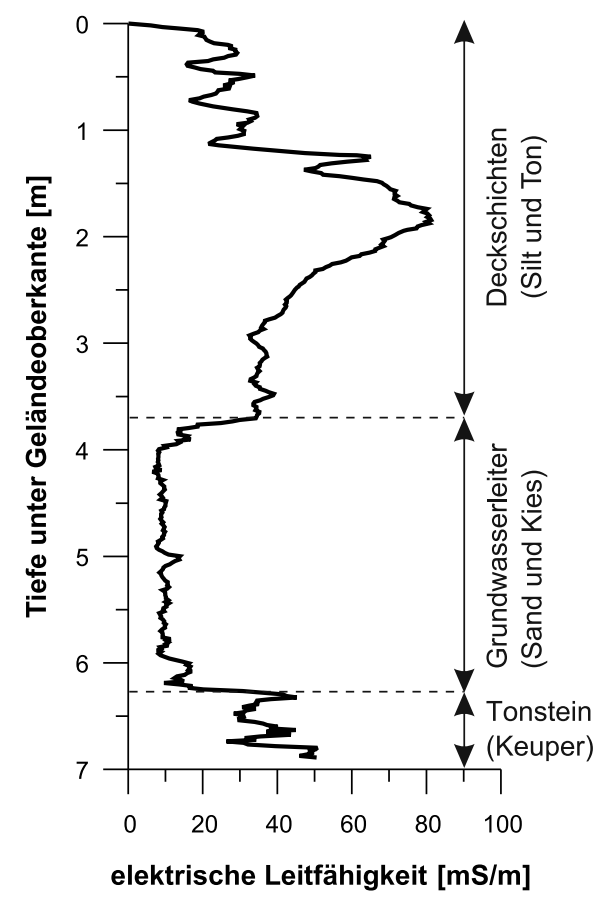

b) DP-Leitfähigkeitslog PM6.4/M15.5

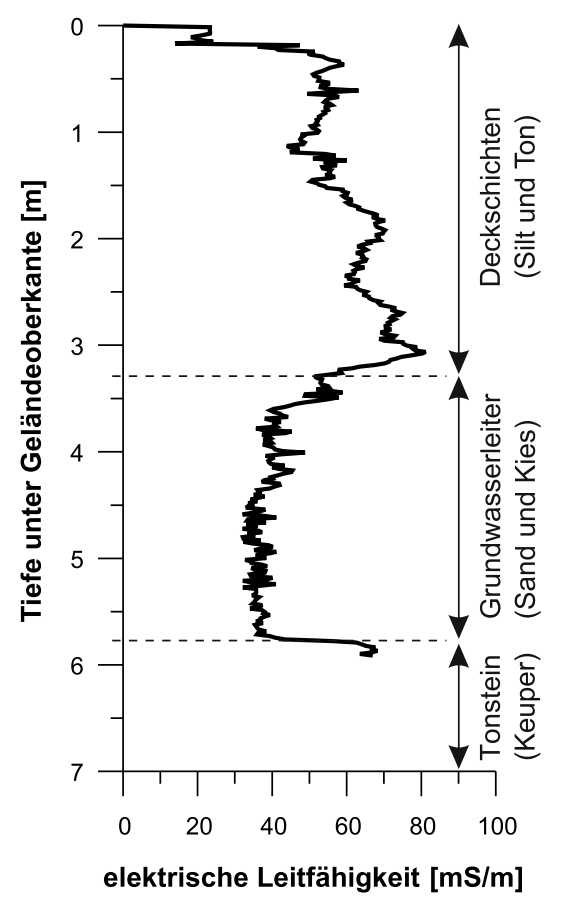

file, die jeweils vor der Installation der 1“-, 2“- und Multikammerbrunnen aufgenommen wurden, hat gezeigt, dass die Variation in der Mächtigkeit des Grundwasserleiters 0,5 $\mathrm{m}$ nicht übersteigt.

\section{Multilevel-Slug-Tests}

Die Durchführung von Multilevel-Slug-Tests ermöglicht die Bestimmung der vertikalen Änderungen der hydraulischen Leitfähigkeit. Die Slug-Tests wurden pneumatisch initiiert um Störungen (Rauschen), die durch das Injizieren/Entnehmen von Wasser/Verdrängungskörper zu Beginn der Aufzeichnung entstanden wären, zu vermeiden. Die Druckaufnehmer in der Wassersäule wurden relativ nahe zur Wasseroberfläche positioniert. Dieses Vorgehen vermeidet Probleme, die darauf beruhen, dass die aufgezeichneten Druckänderungen von der Lage des Druckaufnehmers in der Wassersäule beeinflusst werden (Butler et al. 2003). Die aufgezeichneten Druckänderungen wurden bezogen auf die maximale Druckänderung normiert. Dabei wurde die maximale Druckänderung mit einem zweiten Druckaufnehmer, der in der Luftsäule installiert wurde, bestimmt.

Abbildung 3a zeigt hydraulische Durchlässigkeitsbeiwerte, die in sieben verschiedenen Tiefen verteilt über die gesamte Mächtigkeit des Grundwasserleiters im Brunnen P0/M25 mit Multilevel-Slug-Tests ermittelt wurden. Hierfür wurde ein Doppelpackersystem verwendet, mit dem
0,25 m lange Filterstreckenabschnitte hydraulisch voneinander isoliert werden können. Die hydraulischen Durchlässigkeitsbeiwerte für die einzelnen Abschnitte variieren über eine Größenordnung, zwischen $2 \cdot 10^{-4} \mathrm{~m} / \mathrm{s}$ und $2 \cdot 10^{-3} \mathrm{~m} / \mathrm{s}$. Diese Werte sind typisch für einen Sand- und Kiesgrundwasserleiter (Fetter 1994). Die niedrigsten Werte mit $2 \cdot 10^{-4} \mathrm{~m} / \mathrm{s}$ wurden am oberen Rand des Grundwasserleiters gemessen. Mit zunehmender Tiefe erhöhte sich die hydraulische Durchlässigkeit bis auf $2 \cdot 10^{-3} \mathrm{~m} / \mathrm{s}$ am unteren Rand des Grundwasserleiters.

In Abbildungen 3b-3d sind Multilevel-Slug-Tests, die mit einer Frequenz von $10 \mathrm{~Hz}$ in einer Tiefe von 5,1 m und 4,1 $\mathrm{m}$ aufgezeichnet wurden, und die dazugehörigen Typkurven basierend auf den Lösungen von Butler (1998) und Hvorslev (1951) abgebildet. Die Druckkurve, die in einer Tiefe von 5,1 m aufgezeichnet wurde, zeigt ein leicht oszillierendes Verhalten, das typisch für Bereiche mit höherer hydraulischer Durchlässigkeit ist. Druckkurven die am oberen Rand des Grundwasserleiters, in einem Bereich mit geringer hydraulischer Durchlässigkeit, aufgezeichnet wurden, sind stark gedämpft und weisen kein oszillierendes Verhalten auf (z. B. Abb. 3c). Diese Druckkurven können auch mit einem Geradlinienverfahren (z. B. Hvorslev 1951) verlässlich ausgewertet werden. Abbildung 3c-d zeigt einen Vergleich zwischen den Lösungen von Butler (1998) und Hvorslev (1951). Die ermittelten Werte für die hydraulische Durchlässigkeit unterscheiden sich hierbei nur minimal. 
Abb. 3 (a) Darstellung der Ergebnisse der MultilevelSlug-Tests, die am Brunnen P0/M25 durchgeführt wurden. (b-d) Darstellung der Abweichung des Ruhewasserspiegels $\left(H(t) / H_{0}\right)$, logarithmisch aufgetragen als Funktion der Zeit für drei Slug-Tests, die im Brunnen $\mathrm{P} 0 / \mathrm{M} 25$ in drei verschiedenen Tiefen durchgeführt wurden

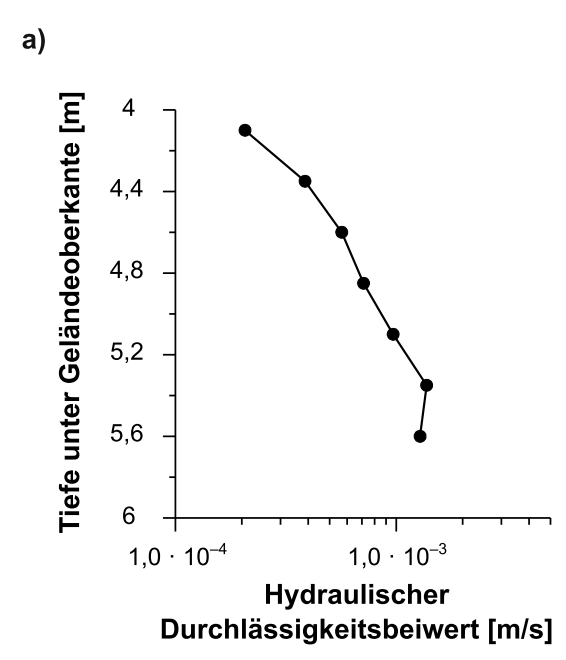

c)

Slug-Test ausgewertet mit der Lösung von Butler (1998)

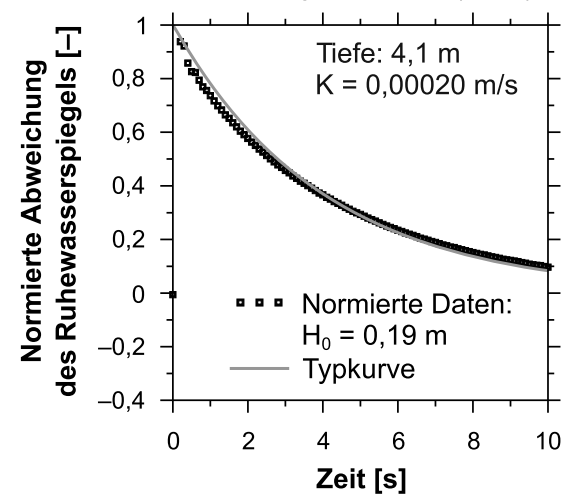

b) Slug-Test ausgewertet mit der Lösung von Butler (1998)

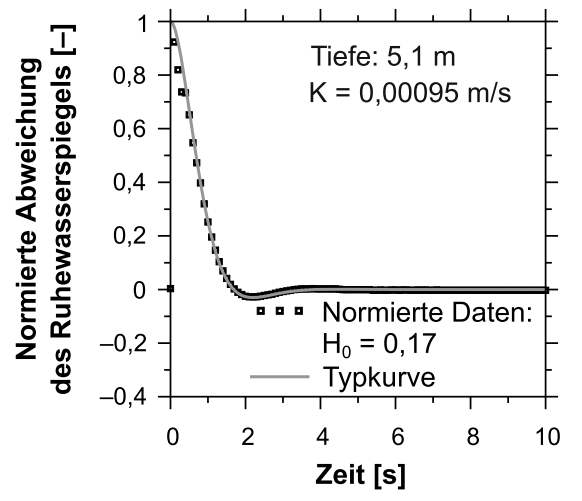

d)

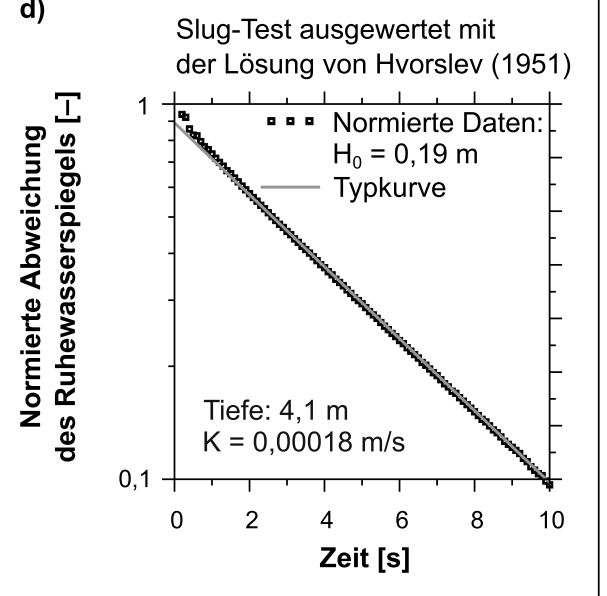

Die dargestellten Ergebnisse zeigen deutlich, dass Multilevel-Slug-Tests sehr gut geeignet sind, um die vertikale Variation der hydraulischen Durchlässigkeit in einem Lockergesteinsgrundwasserleiter in unmittelbarer Nähe des Brunnens zu bestimmen.

\section{Multilevel-Kurzzeitpumpversuche}

Um neben den vertikalen auch laterale Änderungen der hydraulischen Parameter zu bestimmen wurden Kurzzeitpumpversuche unter Verwendung einer tomographischen Messanordnung zwischen einem 2“-Brunnen (P0/M25) und einem Multikammerbrunnen (PM5/M17.5) durchgeführt. Der Multikammerbrunnen basiert auf dem ContinuousMultichannel-Tubing (CMT)-System (Einarson \& Cherry 2002). Dieses System, das ursprünglich für die tiefenorientierte Probennahme entwickelt wurde, besteht aus einem Rohr mit sieben durchgehenden, voneinander getrennten Kanälen bzw. Kammern mit einem Durchmesser von ca. $0,014 \mathrm{~m}$, die wabenförmig angeordnet sind. Vor dem Einbau wurden in die einzelnen Kammern in verschiedenen Tiefen 0,08 m lange Öffnungen gefräst, die mit einem Sandfilter abgedeckt wurden. Diese Konstruktion ermöglicht die Messung der Wasserspiegeländerungen in verschiedenen Tiefen des Grundwasserleiters. Unter Verwendung eines Doppelpackersystems wurden nun in Analogie zu den MultilevelSlug-Tests jeweils 0,25 m lange Filterstreckenabschnitte im 2“-Brunnen P0/M25 hydraulisch voneinander isoliert. An insgesamt fünf Abschnitten wurden Kurzzeitpumpversuche durchgeführt. Bei jedem Kurzzeitpumpversuch wurde die Druckänderung sowohl im Pumpabschnitt im Brunnen P0/M25 als auch in sechs unterschiedlichen Tiefen im benachbarten Multikammerbrunnen PM5/M17.5 mit einer Frequenz von $50 \mathrm{~Hz}$ aufgezeichnet. Der Abstand zwischen dem Pumpbrunnen P0/M25 und dem Beobachtungsbrunnen PM5/M17.5 beträgt $9 \mathrm{~m}$ (Abb. 1). Somit wurden insgesamt 30 (5 - 6) Absenkungskurven aufgezeichnet. In der zentralen Röhre des Multikammerbrunnens wurde keine Druckänderung aufgezeichnet, da dies nur eine eingeschränkte hydraulische Verbindung zum Grundwasserleiter aufwies. Durch die Variation der Pumpintervalle und das gleichzeitige Auf- 
zeichnen der Druckabsenkungskurven in sechs unterschiedlichen Tiefen wurde so ein Signalmuster erzeugt, das mit den Strahlwegen eines seismisch tomographischen Experiments vergleichbar ist.

Auswertung basierend auf analytischen Lösungen

Für die analytische Auswertung der Kurzzeitpumpversuche wurde auf das Geradlinienverfahren von Cooper \& Jacob (1946) zurückgegriffen. Diese Lösung kann für die Auswertung von Pumpversuchen in einem gespannten, homogenen und isotropen Grundwasserleiter herangezogen werden. Die Lösung basiert auf der Annahme, dass die Brunnen über die ganze Mächtigkeit des Grundwasserleiters verfiltert sind. Die Lösung kann aber auch für einen teilweise verfilterten Brunnen angewendet werden, wenn der Beobachtungsbrunnen in einer Entfernung von $1,5 b \sqrt{K_{h} / K_{v}}$ vom Pumpbrunnen steht (Hantush 1964). Der Parameter $b$ beschreibt die Mächtigkeit des Grundwasserleiters und $K_{h}$ und $K_{v}$ die horizontale bzw. vertikale hydraulische Durchlässigkeit. Brauchler et al. (2010) zeigen basierend auf einer hydraulisch-tomographischen Inversion von Interferenz-Slug-Tests, dass in unmittelbarer Nähe zum Brunnen P0/M25 die horizontale hydraulische Durchlässigkeit um den Faktor 5-8 größer ist als die vertikale hydraulische Durchlässigkeit. Bei einer durchschnittlichen Mächtigkeit des Grundwasserleiters von $2 \mathrm{~m}$ und einer angenommenen Anisotropie der hydraulischen Durchlässigkeit von 8 sollte der Abstand zwischen Pump- und Beobachtungsbrunnen nach Hantush (1964) nicht kleiner als 8,48 m sein. Damit sind alle Voraussetzungen erfüllt, um die Kurzzeitpumpversuche mit dem von Cooper \& Jacob (1946) entwickelten Geradlinienverfahren auszuwerten.

Abbildungen $4 \mathrm{~b}-4 \mathrm{f}$ zeigen fünf aufgezeichnete Absenkungskurven, die in unterschiedlichen Tiefen im Beobachtungsbrunnen PM5/M17.5 des Grundwasserleiters aufgezeichnet wurden, und die dazugehörigen Regressionen. Das Geradlinienverfahren wurde nur für Werte rechts von der horizontal eingezeichneten gestrichelten Linie angewendet, um das Minimum-Zeitkriterium einzuhalten (Cooper \& Jacob 1946). Das Minimum-Zeitkriterium wird über die Beziehung $t=\frac{r^{2} S}{0,12 T}$ bestimmt, wobei $r$ den Abstand zwischen Pump- und Beobachtungsabschnitt, $S$ den Speicherkoeffizienten und $T$ die Transmissivität repräsentieren.

Das Testdesign wurde so gewählt, dass der Brunnenspeicher minimiert wurde. In dem hier vorgestellten Beispiel ist der hydraulisch wirksame Pumpabschnitt nur $0,25 \mathrm{~m}$ lang bei einem Innenradius des Doppelpackersystems von 0,0155 m. Weiterhin wird der Bohrlochspeicher durch den Druckaufnehmer (Radius von 0,005 m) und Pumpschlauch minimiert. Numerische Untersuchungen haben gezeigt, dass basierend auf diesem Testdesign (unter Vernachlässigung von Volumen des Druckaufnehmers und Pumpschlauchs), der Bohrlochspeicher keinen Einfluss auf die Bestimmung der hydraulischen Parameter, mit analytischen Lösungen und laufzeitbasierten Inversionsansatz, hat (Hu et al. 2011).

Bei allen fünf Absenkungskurven ist der Pump- und Beobachtungsabschnitt in gleicher Tiefe und der horizontale Abstand zwischen Pump- und Beobachtungsabschnitt beträgt für alle fünf Absenkungskurven $9 \mathrm{~m}$ und übersteigt den nach Hantush (1964) berechneten Minimalabstand um 0,5 m. Die Tiefenangaben in Abbildung 4 beziehen sich jeweils auf die mittlere Tiefe des Pump- bzw. Beobachtungsabschnitts. Die ermittelten Werte für die hydraulische Durchlässigkeit und den spezifischen Speicherkoeffizienten sind in Abbildung 4a als Funktion der Tiefe aufgezeichnet. Die ermittelten Werte für die hydraulische Durchlässigkeit und den spezifischen Speicherkoeffizienten zeigen nur eine geringe Dynamik und variieren zwischen $1,6 \cdot 10^{-3} \mathrm{~m} / \mathrm{s}$ und $2,2 \cdot 10^{-3} \mathrm{~m} / \mathrm{s}$ bei der hydraulischen Durchlässigkeit und zwischen $1,2 \cdot 10^{-4} 1 / \mathrm{m}$ und $2,0 \cdot 10^{-4} 1 / \mathrm{m}$ beim spezifischen Speicherkoeffizienten. Im Vergleich zu den Ergebnissen der Multilevel-Slug-Tests können, basierend auf der Auswertung der Multilevel-Kurzzeitpumpversuche mit der von Cooper \& Jacob (1946) entwickelten Lösung, keine vertikale und lateralen Änderungen in den hydraulischen Eigenschaften des Grundwasserleiters abgeleitet werden. Die ermittelten Werte liegen aber in dem Bereich die mit Multilevel-Cross-Well-Slug-Tests ermittelt wurden. In $\mathrm{Hu}$ (2011) werden die hydraulischen Daten, die am Testfeld Stegemühle ermittelt wurden, im Detail dargestellt.

Auswertung mit dem Ansatz der

hydraulisch-tomographischen Laufzeitinversion

Die tomographische Laufzeitinversion von hydraulischen Tests basiert auf der Transformation der instationären Grundwasserströmungsgleichung in die Wellengleichung unter Verwendung eines asymptotischen Ansatzes (Virieux et al. 1994). Diese Transformation ermöglicht den Einsatz von Ray-Tracing- und Particle-Tracking-Algorithmen für die Inversion. Diese Methoden sind sehr recheneffizient und ermöglichen die Inversion großer Datensätze auf einem herkömmlichen PC. Bei der Transformation der Grundwasserströmungsgleichung in die Wellengleichung wird von einer räumlichen Diffusivitätsverteilung im Untergrund ausgegangen, die sich nicht sprunghaft sondern stetig ändert. Vasco et al. (2000) und Brauchler et al. (2007) haben jedoch gezeigt, dass Parametervariationen über mehrere Größenordnungen hinweg rekonstruiert werden können.

Die Inversion beruht dabei auf der Lösung des folgenden Laufzeitintegrals, das den Zusammenhang zwischen der Laufzeit der maximalen Änderung eines aufgezeichneten instationären hydraulischen Signals und der Diffusivität des 


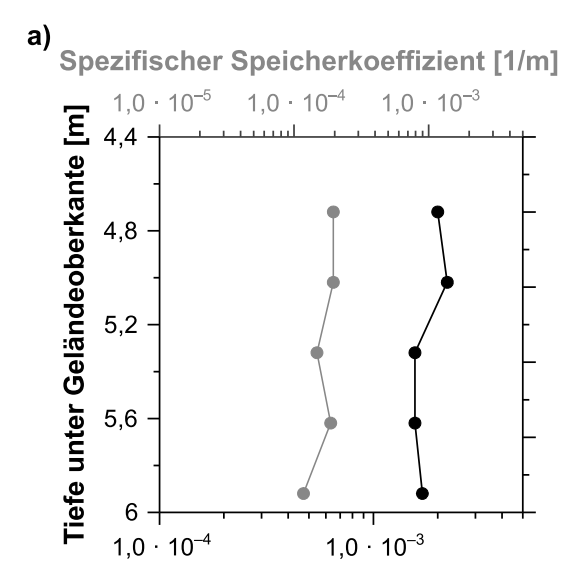

Hydraulischer Durchlässigkeitsbeiwert [m/s]

d)

Kurzzeitpumpversuch ausgewertet mit der Lösung von Cooper \& Jacob (1946)

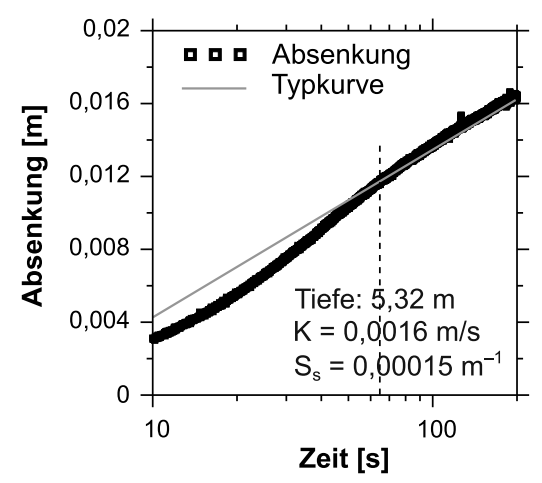

b)

Kurzzeitpumpversuch ausgewertet mit der Lösung von Cooper \& Jacob (1946)

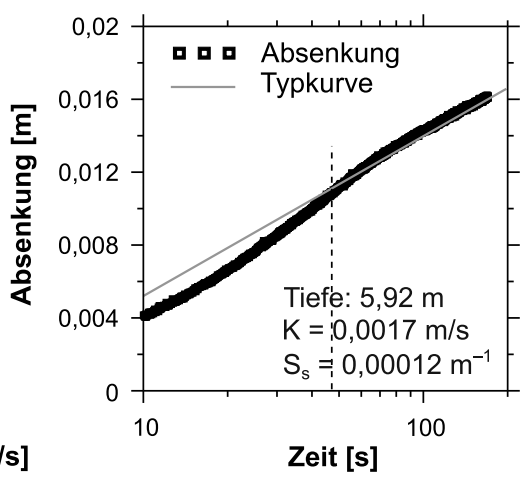

e) Kurzzeitpumpversuch ausgewertet mit der Lösung von Cooper \& Jacob (1946)

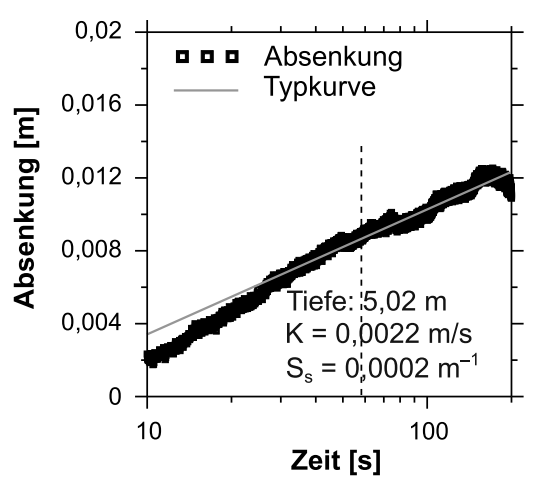

c) Kurzzeitpumpversuch ausgewertet mit der Lösung von Cooper \& Jacob (1946)

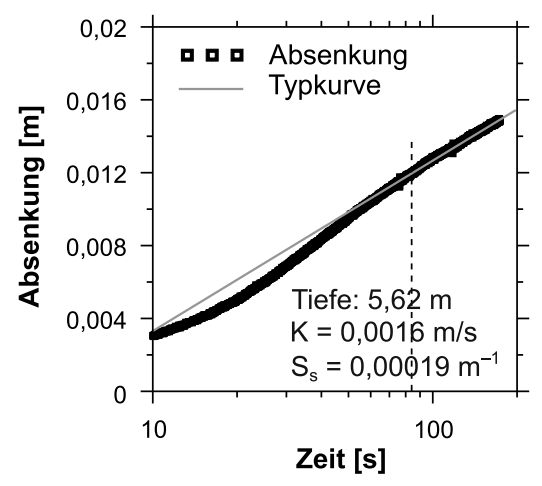

f) Kurzzeitpumpversuch ausgewertet mit der Lösung von Cooper \& Jacob (1946)

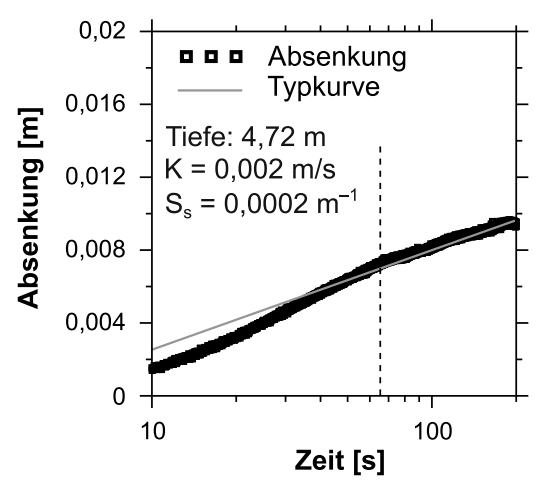

Abb. 4 (a) Darstellung der Ergebnisse der Kurzeitpumpversuche, die zwischen dem 2“-Brunnen P0/M25 und dem Multikammerbrunnen PM5/M17.5 in fünf verschiedenen Tiefen durchgeführt wurden. (b-f) Darstellung der Absenkungskurven, logarithmisch aufgetragen als Funktion der Zeit für fünf Kurzzeitpumpversuche

untersuchten Systems beschreibt (Vasco et al. 2000, Kulkarni et al. 2001)

$$
\text { [1] } \sqrt{t_{\text {peak }}\left(x_{2}\right)}=\frac{1}{\sqrt{6}} \int_{x_{1}}^{x_{2}} \frac{d s}{\sqrt{D(s)}}
$$

Hierbei ist $t_{\text {peak }}$ die Laufzeit der maximalen Änderung eines Drucksignals, das sich vom Pumpbrunnenabschnitt $x_{1}$ (Quelle) zum Beobachtungsabschnitt $x_{2}$ (Empfänger) entlang des Weges $s$ ausbreitet. $D(s)$ ist die Diffusivität (Quotient aus hydraulischer Durchlässigkeit und spezifischem Speicherkoeffizienten) als Funktion entlang des Ausbreitungsweges $s$. Dieses Laufzeitintergral ist nur für eine Impuls-Quelle (z. B. Slug-Test), die mit einer DiracFunktion mathematisch beschrieben werden kann, gültig. Für die Inversion eines Pumpversuchssignals, das mit einer Heaviside-Funktion mathematisch beschrieben werden kann, muss die Heaviside-Funktion in eine DiracFunktion überführt werden. Vasco et al. (2000) haben gezeigt, dass das Druckantwortsignal einer Heaviside-Quelle in ein Druckantwortsignal einer Dirac-Quelle transformiert werden kann, indem man die 1. Ableitung nach der Zeit bestimmt. In Abbildung 5 wird solch eine Transformation für das Druckantwortsignal, das zwischen Pumpbrunnen P0/M25 und dem Beobachtungsbrunnen PM5/M17.5 aufgezeichnet wurde, gezeigt. Für die Ermittlung der 1. Ableitung wurden zuerst eine Wavelet-Rauschunterdrückung und anschließend eine Polynomanpassung durchgeführt. Es ist ebenfalls zu beachten, dass jeder Punkt, der in Abbildung 5 dargestellten Absenkungskurve, größer Null ist. Die mittlere Tiefe des Pumpintervalls befindet sich 5,92 $\mathrm{m}$ und das Beobachtungsintervall 5,62 m unter der Geländeoberkante.

Brauchler et al. (2003) haben Gleichung 1 durch die Einführung eines Transformationsfaktors, der es ermöglicht, neben der Peak-Laufzeit $t_{\text {peak }}$ jede weitere Laufzeit $\mathrm{zu}$ invertieren, weiterentwickelt:

[2] $\sqrt{t_{\alpha, d}}=\frac{1}{\sqrt{6 f_{\alpha, d}}} \int_{x_{1}}^{x_{2}} \frac{d s}{\sqrt{D(s)}}$. 


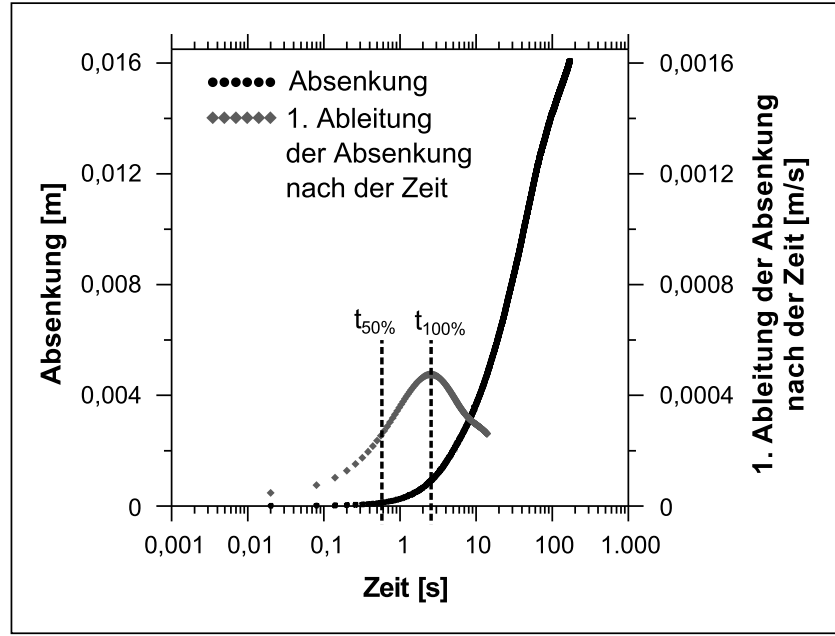

Abb. 5 Darstellung der Absenkung und der 1. Ableitung nach der Zeit für einen Kurzeitpumpversuch, der zwischen dem 2"-Brunnen P0/M25 und dem Multikammerbrunnen PM5/M17.5 durchgeführt wurde. Die Zeit $t_{50} \%$ entspricht der Laufzeit, wenn $50 \%$ der maximalen Steigung des Absenkungssignals erreicht wurde. Diese Zeit wurde für die Laufzeitinversion verwendet.

Hierbei repräsentiert $t_{\alpha, d}$ die Laufzeit eines infinitesimal kleinen Abschnitts eines Signals, das sich vom Pumpbrunnenabschnitt $x_{1}$ (Quelle) zum Beobachtungsabschnitt $x_{2}$ (Empfänger) entlang des Weges $s$ ausbreitet, und $f_{\alpha, d}=$ $t_{\text {peak }} / t_{\alpha, d}$ den dazugehörigen Transformationsfaktor. Der tiefgestellte Index $\mathrm{d}_{\mathrm{d}}$ beschreibt eine Dirac-Funktion. Gleichung 2 ermöglicht nun, basierend auf dem eingeführten Transformationsfaktor, jede beliebige Laufzeit $t_{\alpha, d}$ mit der Diffusivitätsverteilung zwischen Test- und Beobachtungsintervall in Beziehung zu setzen. Hierbei ist der Transformationsfaktor folgendermaßen definiert:

[3] $f \alpha, d=-W\left(-\frac{\left(\frac{h_{d}(r, t)}{h_{d}\left(r, t_{\text {peak }}\right)}\right)^{\frac{2}{3}}}{e}\right)$.

$W$ steht für Lamberts $W$ function und $h_{d}(r, t)$ beschreibt das hydraulische Potenzial als Funktion von Ort und Zeit. Die Herleitung des Transformationsfaktors wird in Brauchler et al. (2003) im Detail beschreiben.

Das Inversproblem erfordert die Lösung eines Gleichungssystems, das sich aus der Diskretisierung von Gleichung 2 für alle betrachteten Ausbreitungswege ergibt. Hierfür kommt der SIRT-Algorithmus (Simultaneous Iterative Reconstruction Technique), der ursprünglich für die seismische und die Radarlaufzeittomographie entwickelt wurde (Gilbert 1972), zur Anwendung. Die Inversion von instationären Druckantwortsignalen basierend auf dem SIRTAlgorithmus wird in Brauchler et al. (2007) beschrieben.

Bei der seismischen oder Radarlaufzeittomographie weisen die rekonstruierten Geschwindigkeitsfelder Änderungen auf, die $30 \%$ nicht überschreiten. Bei der Rekonstruktion von Diffusivitätsfeldern können die Geschwindig- keitsunterschiede hingegen mehrere Größenordnungen betragen. Um den großen Geschwindigkeitsunterschieden bei der Inversion von transienten Druckantwortsignalen Rechnung zu tragen, wurden Ray-Tracing-Techniken angewendet, die es erlauben, die Druckausbreitung entlang von gekrümmten Trajektorien (Ausbreitungspfade) zu berechnen. Ray-Tracing-Techniken verbessern das Inversionsergebnis bei der Rekonstruktion von großen Geschwindigkeitsunterschieden im Vergleich zu Geradstrahlansätzen (z. B. Peterson et al. 1985). Die Ausbereitung des Druckantwortsignals, das von den berechneten Trajektorien abgebildet wird, folgt dem Fermatschen Prinzip. Aus diesem Grund folgen frühe Laufzeiten des aufgezeichneten Druckantwortsignals, z. B. die Laufzeit $t_{50} \%$ wenn 50\% der maximalen Druckänderung erreicht wird, den schnellsten Ausbreitungswegen zwischen Test- und Beobachtungsabschnitt (Abb. 5). Die schnellsten Ausbreitungswege entsprechen gewöhnlich bevorzugten Fliesspfaden. Im Gegensatz dazu charakterisieren späte Laufzeiten eher das integrale Verhalten des untersuchten Mediums, da die Druckänderung große Teile des zu untersuchenden Mediums erfasst hat. Dieses Verhalten haben Cheng et al. (2009) mithilfe einer numerischen Fallstudie verifiziert. Für die im folgenden Abschnitt vorgestellten Inversionsergebnisse wurde die Laufzeit $t_{50 \%}$, wenn $50 \%$ der maximalen Druckänderung erreicht wurde, verwendet. Diese Laufzeit stellt einen Kompromiss zwischen Datenqualität, d. h. Vermeidung von einem schlechten Signal-RauschVerhältnis, und den Erkenntnissen von Cheng et al. (2009) und Brauchler et al. (2010), dass frühe Laufzeiten besser geeignet sind um hydraulische Heterogenitäten aufzulösen, dar. Als Startmodell für die Inversion wurde eine homogene Parameterverteilung, basierend auf dem arithmetischen Mittelwert der gemessenen Druckantwortsignale, gewählt.

Inversionsergebnisse und Methodenvergleich

Für die Inversion wurden insgesamt Laufzeiten von 30 Druckantwortfunktionen simultan invertiert. Die Rechenzeit für die Inversion betrug weniger als eine Minute auf einem PC, ausgestattet mit einer 3,2 GHz-CPU. In Abbildung 6 wird die rekonstruierte Diffusivitätsverteilung (Diffusivitätstomogramm) zwischen dem Pumpbrunnen P0/M25 und dem Beobachtungsbrunnen PM5/M17.5 gezeigt. Diffusivitätswerte variieren über eine Größenordnung, zwischen 1 und $10 \mathrm{~m}^{2} / \mathrm{s}$. Die niedrigsten Werte wurden am oberen Rand des Grundwasserleiters gemessen. Mit zunehmender Tiefe erhöht sich die Diffusivität auf bis zu $10 \mathrm{~m}^{2} / \mathrm{s}$ am unteren Rand des Grundwasserleiters. Diese Verteilung stimmt mit der vertikalen Variation der hydraulischen Durchlässigkeit, die mit den Multilevel-Slug-Tests ermittelt wurde, überein. Mit beiden Verfahren wurden die höchsten Werte für Diffusivität bzw. hydraulische Durchlässigkeit am unteren Rand des Grundwasserleiters ermittelt und die niedrigsten am oberen Rand des Grundwasserleiters. 


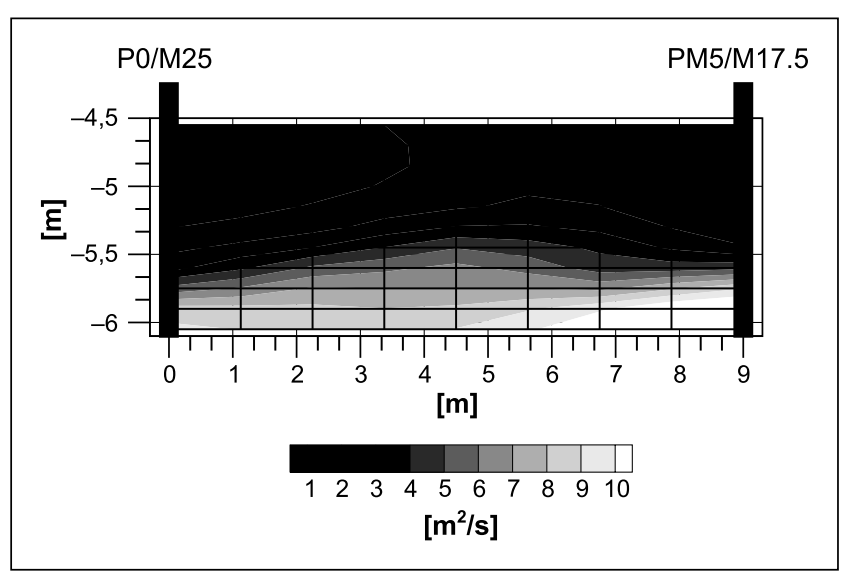

Abb. 6 Darstellung des Diffusivitätstomogramms, das für den Bereich zwischen dem 2"-Brunnen P0/M25 und dem Multikammerbrunnen PM5/M17.5 rekonstruiert wurde

Die berechneten Diffusivitätswerte, basierend auf den Werten der hydraulischen Durchlässigkeit und des spezifischen Speicherkoeffizienten, die mit dem Geradlinienverfahren von Cooper \& Jacob (1946) ermittelt wurden, variieren zwischen $8,4 \mathrm{~m}^{2} / \mathrm{s}$ und $14,2 \mathrm{~m}^{2} / \mathrm{s}$. Diese Werte stimmen mit den Werten, die mit dem laufzeitbasierten Inversionsverfahren am unteren Bereich des Grundwasserleiters rekonstruiert wurden, überein. Das bedeutet, dass die mit analytischen Methoden ermittelten Werte durch die hochdurchlässige Zone am unteren Rand des Grundwasserleiters dominiert werden. Diese Schlussfolgerung stimmt mit den Beobachtungen von Diem et al. (2010) überein, die bei Untersuchungen in einem alluvialen Schotter-Grundwasserleiter festgestellt haben, dass die Pumpversuchsergebnisse die hydraulischen Eigenschaften der hochdurchlässigen Zonen widerspiegeln.

Durch die Kombination der hydraulischen Laufzeittomographie mit weiteren Inversionsverfahren ist es möglich neben der Diffusivitätsverteilung zusätzlich den hydraulischen Durchlässigkeitsbeiwert und spezifischen Speicher zu rekonstruieren. Das Potenzial der Kombination der hydraulischen Laufzeittomographie mit komplementären Inversionsverfahren wird in Bohling et al. (2002) diskutiert und in Brauchler et al. (2007) sowie Brauchler et al. (2011) anhand von numerischen Datensätze und Feldbeispielen aufgezeigt.

\section{Diskussion und Zusammenfassung}

Ziel dieser Feldstudie war ein Vergleich der laufzeitbasierten tomographischen Inversion von Kurzzeitpumpversuchen mit einer analytischen Auswertung sowie die Diskussion und Bewertung der ermittelten hydraulischen Parameter hinsichtlich ihrer räumlichen Auflösung und Verlässlichkeit.

Die Pumpversuche, in einer tomographischen Messanordnung zwischen einem 2“-Pumpbrunnen und einem
$9 \mathrm{~m}$ entfernten Multikammer-Beobachtungsbrunnen durchgeführt, wurden in einem ersten Schritt mit dem Geradlinienverfahren von Cooper \& Jacob (1946) ausgewertet. Die ermittelten Werte für die hydraulische Durchlässigkeit und den spezifischen Speicherkoeffizienten zeigten nur eine geringe Variabilität. Die Werte variierten zwischen 1,6 $10^{-3} \mathrm{~m} / \mathrm{s}$ und $2,2 \cdot 10^{-3} \mathrm{~m} / \mathrm{s}$ bzw. zwischen $1,2 \cdot 10^{-4} 1 / \mathrm{m}$ und $2,0 \cdot 10^{-4} 1 / \mathrm{m}$. Basierend auf diesen Werten war es nicht möglich, Bereiche mit unterschiedlichen hydraulischen Eigenschaften im Untergrund zu identifizieren. Neben den Kurzzeitpumpversuchen wurden im Pumpbrunnen zusätzlich Multilevel-Slug-Tests durchgeführt, die im Vergleich zu den mit dem Geradlinienverfahren ausgewerteten Kurzzeitpumpversuchen deutliche Variationen in der hydraulischen Durchlässigkeit zwischen $2,0 \cdot 10^{-4} \mathrm{~m} / \mathrm{s}$ und $2,0 \cdot 10^{-3} \mathrm{~m} / \mathrm{s}$ zeigten. Die niedrigsten Werte mit $2,0 \cdot 10^{-4} \mathrm{~m} / \mathrm{s}$ wurden am oberen Rand und die höchsten am unteren Rand des Grundwasserleiters gemessen. Ein Vergleich der Ergebnisse aus den Multilevel-Slug-Tests mit den Daten aus den Kurzzeitpumpversuchen zeigt, dass die aus der analytischen Auswertung der Kurzzeitpumpversuche resultierenden hydraulischen Durchlässigkeitsbeiwerte die hydraulischen Durchlässigkeiten der höher durchlässigen Zone, am unteren Rand des Grundwasserleiters, widerspiegeln.

Eine mögliche Erklärung für die geringe Variabilität der Daten basierend auf der analytischen Auswertung könnte sein, dass die Auswertung auf der Absenkung, die zwischen 40 Sekunden und 200 Sekunden nach Pumpbeginn aufgezeichnet wurde, beruht. Für die tomographische Inversion werden hingegen Laufzeiten kleiner als 10 Sekunden herangezogen. Somit werden die Pumpversuche, bei denen sich Pump- und Beobachtungsabschnitt im geringer durchlässigen Bereich, am oberen Rand des Grundwasserleiters, befinden, offensichtlich schon nach 40 Sekunden nach Beginn des Pumpversuchs vom höher durchlässigen Bereich, am unteren Rand des Grundwasserleiters, dominiert.

Schließlich konnte auch gezeigt werden, dass der neben der analytischen Lösung zur Auswertung der Kurzzeitpumpversuche eingesetzte laufzeitbasierte tomographische Inversionsansatz ein großes Potenzial hinsichtlich einer hohen Auflösung und einer detaillierten räumlichen Zuordnung der Diffusivitätswerte zwischen einzelnen Brunnen aufweist. Dies lässt sich zum Teil mit der tomographischen Messanordnung erklären und zum anderen mit der Eigenschaft, dass die Laufzeit eines instationären hydraulischen Signals von der Diffusivitätsverteilung zwischen Pump- und Beobachtungsabschnitt bestimmt wird (Streltsova 1989). Im Gegensatz dazu wird die gemessene Absenkung im Beobachtungsabschnitt nicht nur von den hydraulischen Eigenschaften des Bereichs zwischen Pump- und Beobachtungsabschnitt, sondern auch von Bereichen außerhalb beeinflusst. Dies erschwert, insbesondere im Falle eines stark heterogenen Untergrunds, die räumliche Zuordnung von hydraulischen Parametern, die mit analytischen Lösungen bestimmt wurden. 
Die Feldstudie verdeutlicht insgesamt, dass der laufzeitbasierte tomographische Auswerteansatz aufgrund der hohen räumlichen Auflösung eine Alternative und Ergänzung zu der herkömmlichen Auswertung von Pumpversuchen darstellt.

Danksagung Die vorliegende Arbeit wurde von der Deutschen Forschungsgemeinschaft im Rahmen des Projektes „High resolution aquifer characterization based on Direct-Push technology: An integrated approach coupling hydraulic and seismic tomography“ (Projektnr. BR3379/1-2) finanziert. Die Autoren möchten sich auch für die Unterstützung durch die Stadtwerke Göttingen und das Amt für Umwelt der Stadt Göttingen bedanken.

\section{Literatur}

Bohling, G.C., Zhan, X., Butler, J.J. Jr., Zheng, L.: Steady shape analysis of tomographic pumping tests for characterization of aquifer heterogeneous. Water Resour. Res. 38(12), 1324 (2002)

Bohling, G.C., Butler, J.J. Jr., Zhan, X., Knoll, M.D.: A field assessment of the value of steady shape hydraulic tomography for characterization of aquifer heterogeneities. Water Resour. Res. 43(5), W05430 (2007)

Boman, G.K., Molz, F.J., Boonec, K.D.: Borehole flowmeter application in fluvial sediments: Methodology, results, and assessment. Ground Water 35(3), 443-450 (1997)

Brauchler, R., Liedl, R., Dietrich, P.: A travel time based hydraulic tomographic approach. Water Resour. Res. 39(12), 1370 (2003)

Brauchler, R., Cheng, J.-T., Dietrich, P., Everett, M., Johnson, B., Liedl, R., Sauter, M.: An inversion strategy for hydraulic tomography: Coupling travel time and amplitude inversion. J. Hydrol. 345(3-4), 184-198 (2007)

Brauchler, R., Hu, R., Vogt, T., Al-Halbouni, D., Heinrichs, T., Ptak, T., Sauter, M.: Cross-well slug interference tests: An effective characterization method for resolving aquifer heterogeneity. J. Hydrol. 384(1-2), 33-45 (2010)

Brauchler, R., Hu, R., Dietrich, P., Sauter, M.: A field assessment of high-resolution aquifer characterization based on hydraulic travel time and hydraulic attenuation tomography. Water Resour. Res. 47, W03503 (2011)

Butler, J.J. Jr.: The Design, Performance, and Analysis of Slug Tests. Lewis Pub., 252 S. (1998)

Butler, J.J. Jr., Bohling, G.C., Hyder, Z., McElwee, C.D.: The use of slug tests to describe vertical variations in hydraulic conductivity. J. Hydrol. 156(1-4), 137-162 (1994)

Butler, J.J. Jr., Garnett, E.J., Healey, J.M.: Analysis of slug tests in formations of high hydraulic conductivity. Ground Water 41(5), 620-630 (2003)

Butler, J.J. Jr., Dietrich, P., Wittig, V., Christy, T.: Characterizing hydraulic conductivity with the Direct-Push permeameter. Ground Water 45(4), 409-419 (2007)

Cheng, J., Brauchler, R., Everett, M.E.: Comparison of early and late travel times of pressure pulses induced by multilevel slug tests. Eng. Appl. Comput. Fluid Mech. 3(4), 529-541 (2009)

Christy, C.D., Christy, T.M., Wittig, V.: A percussion probing tool for the direct sensing of soil conductivity. In: Proceedings of the 8th National Outdoor Action Conference, Westerville, Ohio. National Ground Water Association, S. 381-394 (1994)

Cooper, H.H., Jacob, C.E.: A generalized graphical method for evaluation formation constants and summarizing well-field history. Trans. - Am. Geophys. Union 27(4), 526-534 (1946)
Danielson, R.E., Sutherland, P.L.: Porosity. In: Klute, A. (Hrsg.) Methods of Soil Analysis, Part 1. Physical and Mineralogical Methods; Agronomy Monograph, Bd. 9, American Soc. of Agronomy, S. 443-461 (1986)

Datta-Gupta, A., Kulkarni, K.N., Yoon, S., Vasco, D.W.: Streamlines ray tracing and production tomography: Generalization to compressible flow. Pet. Geosci. 7, 75-86 (2001)

Diem, S., Vogt, T., Hoehn, E.: Räumliche Charakterisierung der hydraulischen Leitfähigkeit in alluvialen Schotter-Grundwasserleitern: Ein Methodenvergleich. Grundwasser 15(4), 241251 (2010)

Dietrich, P., Leven, C.: Direct push technologies. In: Kirsch, R. (Hrsg.) Groundwater Geophysics, S. 321-340. Springer, Berlin (2006)

Dietrich, P., Butler, J.J. Jr., Faiß, K.: A rapid method for hydraulic profiling in unconsolidated formations. Ground Water 46(2), 323-328 (2008)

Einarson, M.D., Cherry, J.A.: A new multilevel groundwater monitoring system using multichannel tubing. Ground Water Monit. Remediat. 22(4), 52-65 (2002)

Fetter, W.: Applied Hydrology. Prentice Hall, Upper Saddle River, 691 S. (1994)

Gee, G.W., Bauder, J.W.: Particle-size analysis. In: Klute, A. (Hrsg.) Methods of Soil Analysis, Part 1. Physical and Mineralogical Methods. Agronomy Monograph, Bd. 9, American Soc. of Agronomy, S. 383-411 (1986)

Gilbert, P.: Iterative methods for three-dimensional reconstruction of an object from projections. J. Theor. Biol. 36(1), 105-117 (1972)

Gottlieb, J., Dietrich, P.: Identification of the permeability distribution in soil by hydraulic tomography. Inverse Probl. 11(2), 353-360 (1995)

Hantush, M.S.: Hydraulic of wells. In: Chow, V.T. (Hrsg.) Advances in Hydroscience. Academic Press, New York, S. 281-442 (1964)

He, Z., Datta-Gupta, A., Vasco, D.W.: Rapid inverse modeling of pressure interference tests using trajectory-based traveltime and amplitude sensitivities. Water Resour. Res. 42, W03419 (2006)

Hvorslev, M.J.: Time lag and soil permeability. In: Ground water observations, U.S. Army Corps of Eng. Waterways Exper. Sta. Bull 36 (1951)

Hu, R.: Hydraulic tomography: a new approach coupling hydraulic travel time, attenuation and steady shape inversions for high-spatial resolution aquifer characterization. Hochschulschrift: Göttingen, Univ., Diss., 116 S. (2011)

$\mathrm{Hu}, \mathrm{R} .$, Brauchler, R., Herold, M., Bayer, P.: Hydraulic tomography analogue study: Coupling travel time and steady shape inversion. J. Hydrol. 409(1-2), 350-362 (2011). doi:10.1016/j.jhydrol. 2011.08.031

Illman, W.A., Liu, X., Craig, A.: Steady-state hydraulic tomography in a laboratory aquifer with deterministic heterogeneity: Multimethod and multiscale validation of hydraulic conductivity tomograms. J. Hydrol. 341(3-4), 222-234 (2007)

Illman, W.A., Liu, X., Takeuchi, S., Yeh, T.J., Ando, K., Saegusa, H.: Hydraulic tomography in fractured granite: Mizunami Underground Research site, Japan. Water Resour. Res. 45, W01406 (2009)

Illman, W.A., Zhu, J., Craig, A.J., Yin, D.: Comparison of aquifer characterization approaches through steady state groundwater model validation: A controlled laboratory sandbox study. Water Resour. Res. 46, W04502 (2010)

Kabala, Z.J.: The dipole flow test: A new single borehole test for aquifer characterization. Water Resour. Res. 29(1), 99-107 (1993)

Klute, A., Dirksen, C.: Hydraulic conductivity and diffusivity: Laboratory methods. In: Klute, A. (Hrsg.) Methods of Soil Analysis, Part 1. Physical Mineralogical Methods. Agronomy Monograph, Bd. 9, American Soc. of Agronomy, S. 687-734 (1986)

Kulkarni, K.N., Datta-Gupta, A., Vasco, D.W.: A streamline approach to integrating transient pressure data into high resolution reservoir 
models. In: SPE European Petroleum Conference, 24-25 October 2000, Paris, France. SPE Journal, 6(3) (2001)

Lessoff, S.C., Schneidewind, U., Leven, C., Blum, P., Dietrich, P., Dagan, G.: Spatial characterization of the hydraulic conductivity using direct-push injection logging. Water Resour. Res. 46, W12502 (2010)

Leven, C., Dietrich, P.: What information can we get from pumping tests? Comparing pumping test configurations using sensitivity coefficients. J. Hydrol. 319(1-4), 199-215 (2006)

Li, W., Nowak, W., Cirpka, O.A.: Geostatistical inverse modeling of transient pumping tests using temporal moments of drawdown. Water Resour. Res. 41(8), W08403 (2005)

Li, W., Englert, A., Cirpka, O.A., Vanderborght, J., Vereecken, H.: 2-D characterization of hydraulic heterogeneity by multiple pumping tests. Water Resour. Res. 43(4), W04433 (2007)

Liu, S., Yeh, T.-C.J., Gardiner, R.: Effectiveness of tomography: Sandbox experiments. Water Resour. Res. 38(4), 000 (2002)

Liu, X., Illman, W.A., Craig, A.J., Zhu, J., Yeh, T.-C.J.: Laboratory sandbox validation of transient hydraulic tomography. Water Resour. Res. 43, W05404 (2007)

Liu, G., Butler, J.J. Jr., Bohling, G.C., Reboulet, E., Knobbe, S., Hyndman, D.W.: A new method for high-resolution characterization of hydraulic conductivity. Water Resour. Res. 45, W08202 (2009)

McDermott, C., Sauter, M., Liedl, R.: New experimental techniques for pneumatic tomographical determination of the flow and transport parameters of highly fractured porous rock samples. J. Hydrol. 278, 51-63 (2003)

Melville, J.G., Molz, F.J., Guven, O., Widdowson, M.A.: Multilevel slug tests with comparison to tracer data. Ground Water 29(6), 897-907 (1991)

Molz, F.J., Morin, R.H., Hess, A.E., Melville, J.G., Guven, O.: The impeller meter for measuring aquifer permeability variations: Evaluation and comparison with other tests. Water Resour. Res. 25(7), $1677-1683$ (1989)

Molz, F.J., Young, S.C.: Development and application of borehole flowmeters for environmental assessment. Log Anal. 34(1), 1323 (1993)

Peursem, D.V., Zlotnik, V.A., Ledder, G.: Groundwater flow near vertical recirculatory wells: Effect of skin on flow geometry and travel times with implications for aquifer remediation. J. Hydrol. 222(14), 109-122 (1999)

Peterson, J.E., Paulsson, B.N.P., McEvilly, T.V.: Application of algebraic reconstruction techniques to crosshole seismic data. Geophysics 50, 1566-1580 (1985)

Rovey, C.W.II., Niemann, W.L.: Discussion of "Relationship between pumping-test and slug-test parameters: Scale effects or artifact?" by Butler, J.J. Jr. and Healy, J.M. in Ground Water 36(2), 305313. Ground Water 36(6), 866-867 (1998)

Schulmeister, M.K., Butler, J.J. Jr., Healey, J.M., Zheng, L., Wysocki, D.A., McCall, W.: Direct-push electrical conductivity logging for high-resolution hydrostratigraphic characterization. Ground Water Monit. Remediat. 23(3), 52-62 (2003)

Straface, S., Yeh, T.-C.J., Zhu, J., Troisi, S., Lee, C.H.: Sequential aquifer tests at a well field. Montalto Uffugo Scalo, Italy. Water Resour. Res. 43, W07432 (2007)
Streltsova, T.: Well Testing in Heterogeneous Formations. Exxon Monographs, 413. Wiley, New York (1989)

Sudicky, E.A., Huyakorn, P.S.: Contaminant migration in imperfect known heterogeneous groundwater systems. US Natl. Rept. Int. Union Geol. Geophys. 1987-1990. Rev. Geophys. 29, 240-253 (1991)

Teutsch, G., Klingbeil, R., Kleineidam, R.: Numerical modeling of reactive transport using aquifer analogue data. In: GQ'98: Groundwater Quality Conference, Tübingen. IAHS Publ. 250, S. 381390 (1998)

Vasco, D.W., Karasaki, K.: Interpretation and inversion of lowfrequency head observations. Water Resour. Res. 42, W05408 (2006)

Vasco, D.W., Keers, H., Karasaki, K.: Estimation of reservoir properties using transient pressure data: An asymptotic approach. Water Resour. Res. 36(12), 3447-3465 (2000)

Vesselinov, V.V., Neumann, S.P., Illmann, W.A.: Three-dimensional numerical inversion of pneumatic cross-hole tests in unsaturated fractured tuff. 1. Methodology. Water Resour. Res. 37(12), 30013017 (2001a)

Vesselinov, V.V., Neumann, S.P., Illmann, W.A.: Three-dimensional numerical inversion of pneumatic cross-hole tests in unsaturated fractured tuff. 2. Equivalent parameters, high-resolution stochastic imaging and scale effects. Water Resour. Res. 37(12), 30193041 (2001b)

Virieux, J., Flores-Luna, C., Gibert, D.: Asymptotic theory for diffusive electromagnetic imaging. Geophys. J. Int. 119(3), 857-868 (1994)

Wu, C.-M., Yeh, T.-C.J., Zhu, J., Lee, T.H., Hsu, N.-S., Chen, C.H., Sancho, A.F.: Traditional analysis of aquifer tests: Comparing apples to oranges? Water Resour. Res. 41, W09402 (2005)

Yeh, T.-C.J., Liu, S.: Hydraulic tomography: Development of a new aquifer test method. Water Resour. Res. 36(8), 2095-2105 (2000)

Young, S.C., Pearson, H.S.: The electromagnetic borehole flowmeter: Description and application. Ground Water Monit. Remediat. 15(2), 138-146 (1995)

Zheng, C., Gorelick, S.M.: Analysis of solute transport in flow fields influenced by preferential flowpaths at the decimeter scale. Ground Water 41(2), 142-155 (2003)

Zlotnik, V.A., Ledder, G.: Theory of dipole flow in uniform anisotropic aquifers. Water Resour. Res. 32(4), 1119-1128 (1996)

Zlotnik, V.A., Zurbuchen, B.R.: Dipole probe: Design and field applications of a single-borehole device for measurements of vertical variations of hydraulic conductivity. Ground Water 36(6), 884893 (1998)

Zlotnik, V., Zurbuchen, B., Ptak, T.: The steady-state dipole-flow test for characterization of hydraulic conductivity statistics in a highly permeable aquifer: Horkheimer Insel site, Germany. Groundwater 39(4), 504-516 (2001)

Zhu, J., Yeh, T.-C.J.: Characterization of aquifer heterogeneity using transient hydraulic tomography. Water Resour. Res. 41, W07028 (2005) 